\title{
The Imaginary Mass Lambda-CDM Model
}

\author{
Jason Cole \\ Department of Physics, Warped Dynamics LLC, Harper Woods, USA
}

Email address

Jcole77@rocketmail.com

\section{To cite this article:}

Jason Cole. The Imaginary Mass Lambda-CDM Model. American Journal of Modern Physics. Vol. 5, No. 1, 2016, pp. 1-14.

doi: 10.11648/j.ajmp.20160501.11

\begin{abstract}
The Lambda-CDM (Cold Dark Matter) standard cosmological model is the generally accepted model of modern cosmology. However, many questions remain such as it doesn't explain what Dark matter or Dark Energy is and what it is made of. Also to be the premier cosmological model it can't explain why we see no antimatter in the universe. This paper attempts to answer those questions and many more by incorporating FTL imaginary mass (Tachyons) into the Lambda-CDM (Cold Dark Matter). Rather than having a matter-antimatter big bang this new cosmological theory uses a big bang pair production of matter and FTL imaginary matter. In so doing it explains the effects of Dark Energy, Dark matter and a host of other cosmological phenomena that the current Lambda-CDM standard cosmological model can't answer. To support this Imaginary Mass Lambda-CDM model theory an electromagnetic experimental set-up is proposed so others can test and verify(indirectly) the existence of Tachyon matter.
\end{abstract}

Keywords: Lambda-CDM, Cosmology, Tachyon, Dark Energy, Dark Matter, Quantum Gravity, String Theory

\section{Introduction}

Traditional cosmology theorize that equal amounts of matter and antimatter were produced in the big bang. However, no one can fully explain where the antimatter went. Second, as the universe evolve there isn't a clear understanding of what Dark Energy or Dark Matter is or what they're made of. Based on

$$
\mathrm{e}=\mathrm{mc}^{2}
$$

energy created matter in pairs. That has been proven in particle accelerators. From astronomical observations we see no large quantities of antimatter in our universe. This paper explores a different type of pair production, not of matter and antimatter but matter and Tachyon matter to determine if Dark Energy, Dark Matter and a host of other cosmological questions can be answered by incorporating imaginary mass. Pair production. In which pair production can come in different pairs and isn't limited to matter-antimatter. Figure 1 is a theoretical model of the structure of a Tachyon created along with spherical particles in a new

$$
\mathrm{e}=\mathrm{mc}^{2}
$$

pair production of matter and Tachyon matter pairs. The energy that produced it was a complex energy containing both real and Tachyon energy. That energy produced equal amounts of matter and Tachyon matter. Theoretically FTL imaginary mass could be categorized as a form of antimatter because it's properties is reverse or opposite of normal matter like traditional antimatter is:

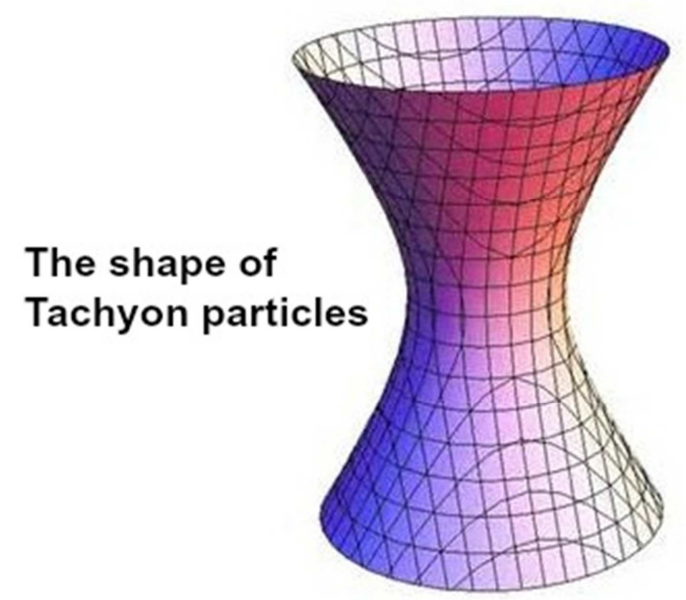

Figure 1. Hypothetically if a spherical particle were to travel faster than light Lorentz contraction would go in reverse. If imaginary mass exists, then the shape of the particle would be a hyperboloid. In which you would have Lorentz expansion giving you a hyperboloid Tachyon shaped particle with negative geometry. The geometry is in reverse like the laws of physics! Going from closed spheres to open end hyperboloids. 


\section{Tachyon Electromagnetic Experiment (T. EM Experiment)}

We can't reproduce tachyon imaginary (negative) mass for Alcubierre warp drive or to make wormhole (Einstein-Rosen Bridge). However, it's possible to reproduce Tachyon negative electromagnetic radiation based on moving charged Tachyons. This can be an indirect way to prove the existence of imaginary mass. The goal is to recreate EM Radiation emitted from moving charged Tachyon matter. The EM waves still travel at the speed of light but it's EM wave structure and properties is reverse compared to normal EM radiation. Based on this new standard cosmological model we now know that the structure of faster than light particles are Hyperboloid in shape. We can attempt to recreate the type of EM radiation that is radiated from tachyon matter. Use a discharging spark or an oscillating molecular dipole where the metal spheres are replaced by hollow Hyperboloid structures. Oscillate those Hyperboloid dipoles to propagate a tachyon electromagnetic wave. The idea is that at sufficiently high energies the electromagnetic waves should exhibits bizarre tachyon EM wave properties. Having reverse electromagnetic wave properties. How high that energy has to be to produce manifestation of Tachyon EM wave is to be determined but it will be in the realm of technological feasibility. Also by colliding a beam of negative EM waves against normal radiation it's possible to create an Electromagnetic wave wormhole. Following show an experimental setup of moving charged Tachyon particles:

\section{Propagation of an Tachyon EM wave}

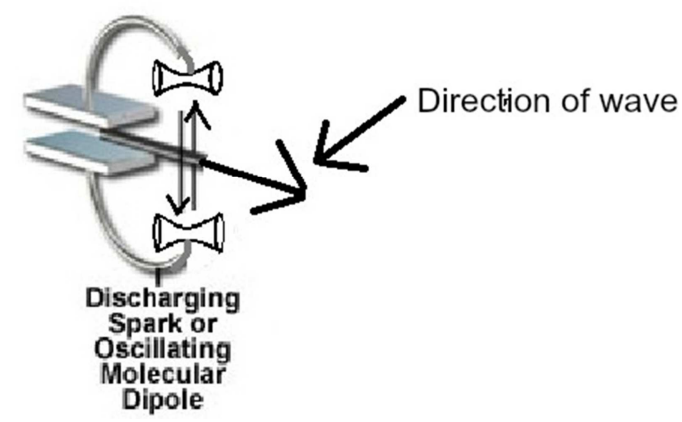

Figure 2. A Tachyon EM experiment aimed at recreating how moving charged imaginary particles generate EM waves. The goal is to generate a reverse structure EM with imaginary physics properties using oscillating molecular Dipoles shaped like hollow hyperboloid structures. The electric field configuration will mimic the electric field of Tachyon particles in hopes of generating a new type of Tachyon EM wave.

It is predicted the EM wave will be an open or hyperbolic configured wave compared to normal EM waves like the Hyperboloid geometry is to the sphere. Also it's possible to create a Tachyon EM field by having a charge hyperboloid $3 \mathrm{~d}$ structure and rotating it (simply adding motion) to produce a Tachyon EM field. This will be more complicated and costly because an ultra-high energy rotating hyperboloid charge structure will have to be built to recreate a Tachyon EM field. Placing a normal EM field close to this Tachyon
EM field could also create a EM wormhole. If the Tachyon EM wave experiment is successful then the complicated and costly Tachyon EM fields can be built. The T. EM experiment is like a geometric shortcut to access FTL physics by imitating the geometry of charged FTL particles and recreating its electromagnetic structure in hope of recreating its reverse physics. The experiment will enable us to go beyond the I.M. cosmological theoretical model to applied high energy Tachyon physics. If this experiment reproduces the Bizarre electromagnetic radiation properties of Tachyon electromagnetic waves, then it will support the validity of this new Imaginary Mass Lambda-CDM model.

Use a Maxwell EM equation for Tachyon EM can give precise mathematics to back up the experiment. Also the math can help build a precise hyperboloid oscillating structures. It is the contour of the Hyperboloid structures that will give raise to the Tachyon EM wave. The contour will determine the shape of hyperboloid Electric field so it can oscillate a Tachyon EM wave. If the Tachyon EM experiment is successful then it can not only transmit Tachyon EM waves (and generate Tachyon EM fields) but it can also serve as a Tachyon receiver antenna. This could open the door to a whole new area of astronomy showing Tachyon radiation from the cosmic background radiation, black holes to any Tachyon EM radiation from the reverse EM spectrum.

The next sections explain how the geometries of Tachyon particles was discovered. Further sections discuss the effects of a quantum entangled imaginary Tachyon universe to our visible universe to explain quantum gravity, Dark Energy, Dark Matter, GRBs and inflation that the current LambdaCDM can't answer.

\section{Explaining How the Geometry of Imaginary Tachyon Particles Was Discovered}

To better understand the physics of a Tachyon base universe we first need to know the quantum mechanical structure of Tachyons. Let's do a thought experiment of an object traveling to the speed of light and beyond and observing its Lorentz transformation to determine the geometry of the particle as it travels faster than light. Hypothetically let's say a sphere accelerate towards $\mathrm{C}$ and then $\mathrm{V}=\mathrm{C}$ and then $\mathrm{V}>\mathrm{C}$ in a thought experiment. The sphere as it travels towards $\mathrm{C}$ will contract but at speeds above $\mathrm{C}$ it will expand. The new geometry of the particle will be a hyperboloid. I call this "Lorentz Expansion". Beyond C the mass becomes imaginary and the physics changes. The focus is on the geometry of the particle at speeds greater than $\mathrm{C}$.

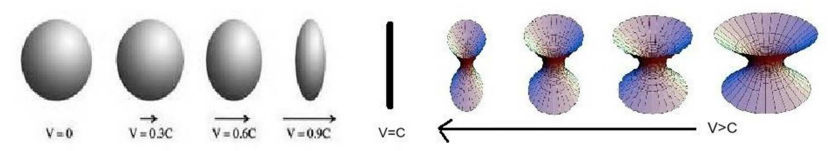

Figure 3. This gives an illustration of Lorentz transformation for objects traveling from slower than light $(V<C)$ to faster than light $(V>C)$. 
Beyond $\mathrm{C}$ you would have an expanding Hyperboloid at different speeds above $\mathrm{C}$ under "Lorentz Expansion" until you reach the maximum velocity above $\mathrm{C}$. The maximum Velocity above $\mathrm{C}$ is the equivalent of an imaginary mass object being at rest. In which there is a limit beyond C. Its speed can't increase forever. It appears that $2 \mathrm{C}$ is the rest speed for tachyon objects were it mirrors $\mathrm{V}=0$ speed objects in our visible universe. In this reverse physics system, the top velocity (2C) would be the rest starting point of objects faster than light. From that top speed they would decelerate towards the speed of light like objects in our visible universe accelerate towards $\mathrm{C}$.

This Lorentz transformation can be compared to the three type of space geometry. Elliptic $(\mathrm{V}<\mathrm{C}$ represents sphere matter), Euclidean $(\mathrm{V}=\mathrm{C}$ where the sphere will become a cylindrical line represents massless particles), Hyperbolic $(\mathrm{V}>\mathrm{C}$ is where it takes on hyperboloid shape represents faster than light imaginary mass particles such as a Tachyon proton). Space curve geometry is a function of speed.
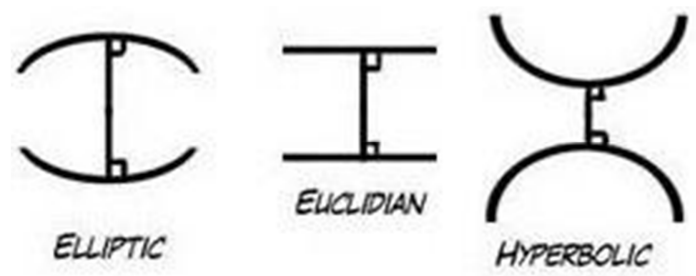

Figure 4. Illustration above gives the quantum geometry of particles from slower than light to faster than light. The geometry of the particles would reverse like the physics.

We can also express imaginary mass mathematically using the Equation

$$
\mathrm{e}^{2}-\mathrm{c}^{2} \mathrm{p}^{2}=\mathrm{m}^{2} \mathrm{c}^{4}
$$

- M squared greater than zero-- protons (spherical shape particles). Traveling slower than the speed of light

- M squared equal zero-- photons (massless particles). Traveling at the speed of light. The shape of massless particles is cylindrical.

- M squared less than zero (hyperboloid shape protons with imaginary mass traveling faster than the speed of light)

Mass, massless \& imaginary mass corresponds to different space geometries. Objects traveling slower than light has Elliptic or spherical geometry, Euclidean geometry represent massless objects such as photons, Hyperbolic geometry represents particles traveling faster than light. In which hyperboloids are the shape of faster than light particles.

\section{The New Big Bang (Matter and Imaginary Matter Creation)}

At the big bang matter and hyperboloid Tachyon imaginary matter was created and not matter and antimatter. Because Tachyon matter has reverse physics of normal matter that it can also fit in the context of anti-matter. It's opposite matter in every sense of the word not just in charge but in geometry and tachyons. The Energy that created this matter and Tachyon mass was truly unique. A complex energy containing real and imaginary parts. Normally

$$
\mathrm{e}=\mathrm{mc}^{2}
$$

creates matter and antimatter. However, the energy in the big bang created matter (slower than light) and imaginary (FTL) matter. Because massless particles travel at the speed of light it does not violate the laws of physics that energy from the big bang could have created both slower than light matter and faster than light imaginary matter (Tachyon) pairs rather than normal matter and antimatter pairs.

\section{e(complex $)=(\mathrm{mi}+\mathrm{m}) \mathrm{c}^{2}$ Matter and imaginary matter creation \\ (5)}

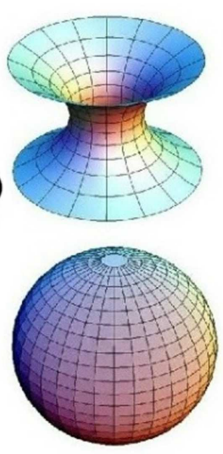

Figure 5. A big bang pair production of matter and Tachyon matter rather than matter-antimatter. In this big bang pair production sphere particles and Tachyon hyperboloid particles were made in pairs like brother and sister twins rather than identical same gender twins.

Just as our universe exploded from a point the imaginary universe exploded from a ring type structure. Imagine the base rim of a hyperboloid exploding. Rather than expanding and accelerating like our universe the imaginary universe is contracting and decelerating from a ring explosion. This imaginary universe is traveling faster than the speed of light with reverse physics. The electromagnetic field, strong force, weak and gravity field will be a negative mirror image of our universe.

The following list the mass-energy conversion equation according to the geometry it is in

$$
\mathrm{e}=\mathrm{mc}^{2}
$$

normal matter and antimatter pair production in our spherically curved universe. Photon can create matter and antimatter pairs. These virtual particles come from the positively curved space vacuum. In positive curved space spherical particles can be set up to be quantum entangled.

$$
\mathrm{ei}=(\mathrm{mi}) \mathrm{c}^{2}
$$

Tachyon matter and anti-Tachyon matter pair production where ei is imaginary energy and (MI) is imaginary mass in the negatively Tachyon universe. Imaginary photon can create Tachyon matter and anti-Tachyon matter pairs. This also applies to virtual Tachyon matter and anti-Tachyon matter. These virtual particles come from the negatively curved space vacuum. In negatively curved space hyperboloid particles has the ability to be quantum entangled. 


$$
\mathrm{e}(\text { complex })=(\mathrm{m}+\mathrm{mi}) \mathrm{c}^{2}
$$

matter and Tachyon matter pair production where e represents complex energy. It can be matter and anti-Tachyon matter pairs or anti matter and Tachyon matter pairs. This type of energy existed at the big bang and inside black holes. Complex photons can give raise to regular and Tachyon photon pairs. Complex EM radiation has a dual wave structure of normal semicircle EM waves and Tachyon hyperbolic waves. In this flat Euclidean space, it can give raise to virtual matter and Tachyon matter pairs from its quantum vacuum energy. In flat curved space complex energy can produce pair particles that are quantum entangled

\section{Connection to String Theory}

The sphere and open end Hyperboloid particles based on a big bang pair production shows a striking correlation with closed and open strings in String theory! Early string theory predicted the Tachyon particle and based on the new big bang pair production it has matter and Tachyon matter pairs. String gas cosmology predicts a dual universe of one expanding and one contracting. Based on this new big bang of matter and Tachyon pair production our matter universe expands and a Tachyon universe will contract. This connection to string theory will be described in more detail later in the paper. It's possible that this deep connection is describing a new form of string theory that goes beyond M-Theory.

\section{The Relativistic Tachyon Particle Symmetry Theory}

Based on the I.M. standard cosmological model all fundamental particles have a tachyon matter partner. Tachyon could also be categorized as a form of antimatter. Tachyon particles are more opposite to normal particles than antiparticles are to normal particles. In which Tachyon particles have opposite particle geometry, travel FTL, time direction, charge, spin, etc. while antimatter just have an opposite charge. Just as normal matter can have antimatter the tachyon matter can have anti-tachyon matter partners. On an overall scale matter and tachyon matter are also a form of matter and antimatter pairs based on "The Tachyon Particle symmetry theory. Particles that travel slower than light have a mirror image partner in the tachyon universe and they are always created in pair. The standard model of particle physics has a Tachyon mirror image particle model. They are Quantum entangled and operate as one system.

The Relativistic Tachyon Particle symmetry theory does not violate Special Relativity because Tachyon matter was created in the big bang as matter-tachyon matter pair creation instead of matter-antimatter.

\section{The Imaginary Atom}

Compared to a spherical proton the Quantum mechanical structure of an imaginary proton consists of a Hyperboloid structure. These imaginary mass protons will make up the mass of an imaginary universe. It's possible that an electron cloud is orbiting around the curved sides of hyperboloid proton in an overall hyperboloid shaped Tachyon cloud surrounding the hyperboloid proton. Image below is an imaginary mass proton atom and Hyperboloid proton-neutron nucleus.

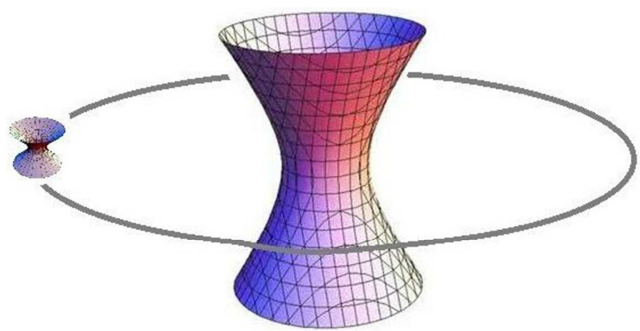

Hyperboloid Nucleus

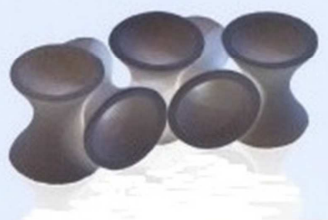

\section{Proton \& Neutron configuration}

Figure 6. The illustration above is a graphical representation of a single Tachyon hyperboloid shape hydrogen atom. The shape of the electric and magnetic fields and their interaction will be reverse compared to normal spherical atoms electromagnetic interactions. Other picture shows possible arrangement of Hyperboloid protons and neutrons. There will be a nuclear strong and weak force for Tachyon nucleus. How the multiple Tachyon electron orbit configuration around that linear atom will be an interesting study. The atoms linear configuration may produce linear large scale macro objects. Planets, stars, etc. will have hyperboloid shape in contrast to our spherical shape planets, moons and stars.

\section{Imaginary Electromagnetic Radiation (Tachyon EMS)}

Just as ordinary radiation has a waving pattern from Radio waves to Gamma rays the EM wave pattern in the Tachyon universe will be a reverse Electromagnetic spectrum (EMS). Hyperboloid electric field shape will give raise to a different EM propagation wave. The Tachyon EM waves still travel at the speed of light but its EM wave structure will be reverse just as a hyperboloid particle is reverse in geometry to a spherical particle. When Tachyon electrons are excited in the orbits of Tachyon atoms they would give off Tachyon photons. These photons would have a completely reverse EM wave structure compared to normal light. Imagine EM field and wave properties in reverse. This new form of radiation would be imaginary EM radiation. The following gives a comparison of normal light to Tachyon light. Both travel at the speed of light but have reverse EM structures like a sphere compared to a hyperboloid. The properties of the Tachyon wave can be determined by Maxwell Equations. Tachyon EM waves are to normal EM waves as Tachyon repulsive gravity is to normal attractive gravity. 


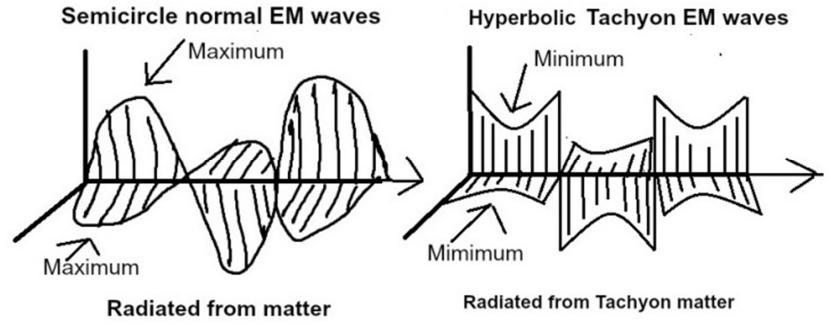

Figure 7. Regular EM can be represented as a harmonic wave of electric and magnetic fields fluctuating orthogonal to each other. The uniform circular motion is represented as a sine function. For the Tachyon EM wave it also represents as a sine wave but a discontinuous harmonic sine wave function of electric and magnetic fields.

Tachyon EM wave discontinuous motion is based on a hyperbolic wave cycle. One side of the hyperbolic parabola is the trough part and the bottom side of the hyperbolic parabola is the trough. Together they form one hyperbolic discontinuous sine wave cycle like a continuous sine wave represent a full circle. One arc of the hyperbolic is 0 to 180 degrees and the other arc is 181 to 360 degrees. Compared to a $2 \mathrm{~d}$ flat circle a hyperbolic is an open 360-degree structure which mean it's not continuous like a circle from 0 to 360 degrees. Like with everything the Tachyon universe is opposite of our visible universe in every way and that includes sine wave functions. One has continuous sine functions and the other has discontinuous sine wave functions. With a hyperbolic structure you can take a pencil and make a continuous 360-degree circle. What would have us a gap between the two arcs. We see that the arc of the outwardly curved hyperboloid should be 180 degrees. The oscillator must be open Hyperboloid so the electric field can have a Hyperboloid configuration to propagate a discontinuous hyperbolic EM wave. Just as spherically charged (electric field) particles radiate EM radiation to give semicircle EM waves, an oscillating charged hyperboloid particle (electric field) would give hyperbolic shaped EM waves. Taking Maxwell Electromagnetic equations but in reverse. It would be a Tachyon version of Maxwell EM equations. The goal of the Tachyon EM experiment is to oscillate hyperboloid shape structures to imitate the Tachyon electric field to create a Tachyon EM wave. The figures above are just drawings but Maxwell EM equations can give a precise picture of the Tachyon EM waves and their properties. Also the math can help build a precise hyperboloid oscillating structures. It is the contour of the electric field shape Hyperboloid structures that will give raise to the Tachyon EM wave. The contour will determine the shape of hyperboloid Electric field so it can propagate a Tachyon EM wave. This Tachyon form of EM radiation is to ordinary light like antigravity is to our normal gravity. You can make a Tachyon transmitter or receiver. Also this technology opens the door to a new wave of Tachyon spectrum applications from Radio to X-ray transmitters or receivers. Applications range from astronomy to medical usages.

\section{Tachyon Trigonometry (Discontinuous Sine Wave) Functions}
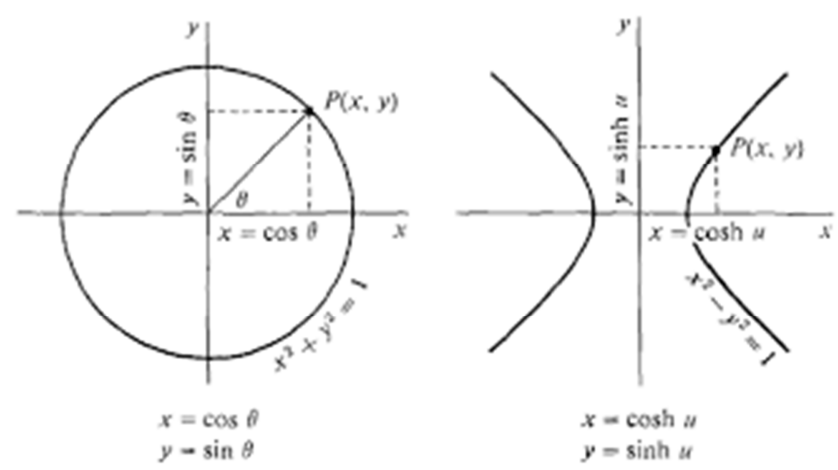

Figure 8. Traditional sine waves represent the EM spectrum and other continuous phenomena in the visible universe. However, for Tachyon EM waves it is based on a discontinuous function. One side of the hyperbolic parabola is the top crest and the other half of the hyperbolic parabola is the bottom trough forming a complete hyperbolic wave analogous to a complete circle for continuous sine waves. One arc of the hyperbolic is 0 to 180 degrees and the other arc is 181 to 360 degrees. Compared to a $2 d$ flat circle a hyperbolic is an open structure which mean it's not continuous. We see that the arc of the outwardly curved hyperboloid should be 180 degrees. The hyperbolic sine wave would be a discontinuous function. Tachyon trigonometry is a new branch of discontinuous hyperbolic sine wave functions that represents the physics of certain waves in the FTL Tachyon universe. This Tachyon discontinuous sine function reversely mirrors our continuous functions. In the Tachyon EM experiment we're using open Hyperboloid dipoles (open and curved outwardly) in an attempt to produce a hyperbolic discontinuous sine waves of EM wave radiation. It's those curved outward sides that gives raise to the discontinuous hyperbolic EM sine wave. The curves must arc outwardly so the hyperbolic shape electric field can produce hyperbolic shape EM waves. The same applies when trying to generate a EM Tachyon EM field.

\section{Tachyon Quantum Mechanics}

The entire quantum mechanics landscape has a tachyon mirror image. A complete picture of an atom it's the visible atoms we see and their tachyon partner. Everything from electron standing waves (or electron clouds), and all other quantum mechanics systems will have a tachyon mirror image including mirror image probability. These reverse mirror systems will balance each other out were the total charge, spin, mass, position, momentum, speed, geometry, etc. will balance out to zero like a balancing scale. Studying Tachyon Q.M. can help advance the understanding of our visible Q.M. landscape in our universe. When you plug in imaginary values to Quantum Field equation it is describing the Tachyon quantum landscape. The interesting idea is that the real and imaginary FTL QM landscapes are quantum entangled operating as one overall quantum system.

The Quantum harmonic Oscillator in our universe also have a Tachyon mirror image partner. Just as our universe can have virtual matter-antimatter pairs so can the Tachyon universe have virtual Tachyon matter and anti-Tachyon pairs. They operate as one overall quantum entangled system and balance out. Flat Euclidean geometry (zero curve space) which is like a mirror between the two different geometries 
can also have a quantum vacuum energy state giving virtual matter-Tachyon matter pairs.

The imaginary universe has the reverse structure of our visible universe. For example, atoms may contain multiple hyperboloid protons and neutrons atoms were multiple electrons orbit around the nucleus. There is a nuclear strong and weak force equivalent for Tachyon nucleus holding them together. In fact, there is a Tachyon standard model of particles for the Tachyon universe that mirrors our particle standard model. Tachyon nucleus have electrons orbiting around them a how those electrons are orbiting the hyperboloid nucleus is yet to be determined. There exists an electromagnetic interaction between the nucleus and the Tachyon electron. How these hyperboloid atoms connect to form molecules and larger structures of matter is yet to be determined? Even though imaginary mass on a large scale has repulsive gravity you still have the Tachyon strong, weak and electromagnetic force holding things together giving things structure. The concentration of these hyperboloid atoms would create a large scale Hyperboloid structure equivalent to a planet, star. In which the shape of moons, planets and stars in an imaginary universe would be hyperboloid in shape, not spherical. Based on the tachyon atomic structure it appears that the atoms bond in linear directions. Only the curved sides tachyon atom may be where the tachyon electron bond with other tachyon atoms. In which the bonding isn't omnidirectional like on slower than light spherical hydrogen atoms. This linear bonding may give raise to the hyperboloid macro structures of planets, moons, stars, etc. Tachyon Quantum Mechanics is based on the idea that it's quantum landscape is quantum entangled to our QM landscape and they balance out acting as one quantum system. That quantum entanglement extends to the macro and cosmological level were the dual universes operate as one universe balancing each other out.

\section{Using Tachyon Imaginary Mass-Energy in Einstein Field Equations}

When referencing to Tachyon physicists have always referred to them traveling but no one has looked at them in the context of gravity. In which imaginary energy-stressmomentum can be used in Einstein Field Equation. Just as Tachyon mass has momentum and energy different than normal matter so would the warping of space of tachyon mass. In this context the imaginary energy-mass-momentum would create negative pressure. Tachyon mass would be imaginary mass-energy substance in Einstein Field equation to produce a type of negative pressure (reverse the positive compressing pressure of typical field equations). Tachyon matter wouldn't curve space like ordinary matter but would have a reverse space warp configuration. It is predicted it would be hyperboloid in shape and correspond to Lorentz transformation of objects traveling faster than the speed of light. How imaginary Hyperboloid atoms configure to form large scale structures such as moons, planets, stars are yet to be determine.
Remember Tachyon atom still use electromagnetism to bond matter together. Nuclei of Tachyon atoms still have its version of the strong and weak force to hold the nucleus together. Below is an image of how tachyon matter-energy might warp space to produce anti-gravity effect on a large scale.

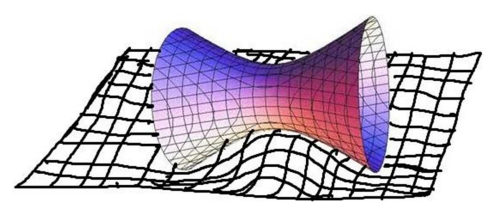

Curving space up like a hill (Anti-gravity)

Figure 9. Illustration above is an image of how Tachyon imaginary energystress-momentum Tensors might warp space to produce anti-gravity. Stronger anti-gravity (higher hills of curvature) will be related to the reduced radius of the hyperboloid and its increase negative density.

Remember the tachyon universe have the reverse of the strong and weak force for the tachyon nuclei. They'll overcome the repulsive nucleus and keep the Tachyon protons and neutrons together. Tachyon proton and electron still interact via electromagnetic force to make larger atoms and attractions. Tachyon matter universe has reverse mirror image laws of physics. Even though gravity is repulsive on a large scale you still have those three other forces (strong, weak and electromagnetism) holding the Tachyon matter together to make large scale structures from planets to galaxies. The orbital mechanics of an anti-gravity system is yet to be determined but they'll be a reverse mirror imagine to our normal gravity orbital systems.

Einstein Field Equation can be expanded to cover the different space curve (gravity) geometries. The Hyperboloidlike manifold is the basis to calculate a negative Riemann curvature tensor. Imagine the hyperboloid standing up vertically \& you're at the equator and the open ends represents the north \& south pole areas. We see that spherelike manifolds can be used for gravity, Hyperboloid-like manifolds for antigravity. A combination of matter and repulsive gravity can be described by a cylinder manifold. It would represent static gravity where it is not pulling space down for gravity nor pushing space up for antigravity. It keeps it in the same place. The Riemann curvature tensor is applicable to the 3 different space curve geometries: spheres, hyperboloids and cylinder representing 3 different gravity states. The 3 different gravity states of space curvature is analogous to orbits were objects spiral in, orbit or spiral out. Likewise, space can warp down for gravity, warp up for repulsive gravity or warp flat or level for static gravity. Static gravity isn't empty or void gravity but a gravity that holds things in the same place rather than attract or repel.

\section{Redefining Black Holes: Matter and Tachyon Matter Combined Stars}

Force of gravity is the product of their masses. The two tachyon imaginary mass would affect each other by anti-gravity effect. The reason why is that the product of their imaginary 
masses would yield a negative sign (repulsive) gravitational force. For black hole it would be the product of matter and imaginary matter. We don't know how matter and imaginary matter will interact but the result will be imaginary rather than real. This gives us the first mathematical description of a black hole because it is where matter and imaginary matter meet at a point of inflection. In which the force of gravity is the product of the matter and imaginary matter product. Matter and Tachyon matter are quantum entangled and meet at a black hole. When mass warp space down (gravity) so do Tachyon matter warp space up (antigravity) based on quantum entanglement. At extreme gravities (black hole) is where the two opposing gravity meet. In which the Tachyon matter is like the complex conjugate of visible matter. This assumption is based on the Relativistic Tachyon Particle symmetry theory were just as quantum mechanical objects have FTL mirror partners so do large scale structures of matter have mirror images structures in the FTL universe. The two opposing masses and their gravity warps are quantum entangled as one system. At extreme gravities they meet at a point of inflection (X-intercept). Mass presents positive pressure, tachyon mass represents negative pressure and the singularity represents Neutral pressure. In terms of Einstein Field Equation Zero pressure is a combination of negative and positive pressure. In which it is not a force that compresses or pulls apart but keeps in the same place. This is where positive curve space fusion with negatively curve space and form a zero curve space analogous protons merging into electrons to form a neutron star. Rather than mass merging together it is space curves merging together. It's like two opposing gravity wells converging to a single point(singularity). Gravity represented as a conjugate and tachyon gravity as its complex conjugate. They meet at a black hole. The following picture show a gravity well and its reverse mirror image gravity well meeting at the $\mathrm{X}$ intercept (black hole).

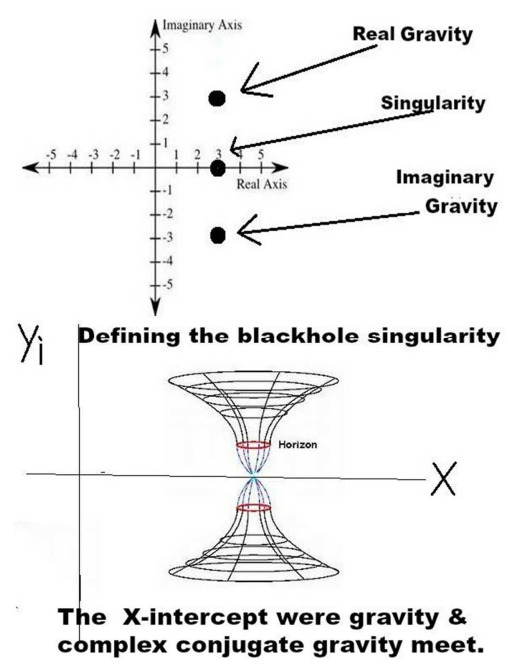

Figure 10. Pictures above show a complex coordinate plane for complex Metric Tensors of Einstein Field Equations for gravity and antigravity formation into a black hole. It's a quantum entangled gravity system. Both gravities are imaginary with respect the complex coordinate plane. Visible matter-gravity is called Real gravity on the graph to differentiate it from Tachyon anti-gravity on the lower part of graph. From a geometric perspective the spherical matter of our universe merged with the hyperboloid shape matter of the Tachyon universe and form cylindrical shaped matter.
The physics of a black hole can be described by a cylinder manifold as it related to Riemann curvature tensor. The spherical slower than light matter in our universe combines with the FTL matter of the Tachyon universe and forms a cylindrical manifold at the speed of light between those two states. From a quantum gravity perspective, the black hole cylindrical manifold would have complex Gravitons. Spin 2 Gravitons plus imaginary spin 2 repulsive Gravitons giving overall complex Gravitons for black holes.

When the two opposing gravities meet at the X-intercept the normal matter black hole will be at its highest attractive strength while the negative mass black hole will be at its lowest repulsive strength. Matter and Tachyon matter can only meet at the General Relativity extremes of black holes or the big bang singularity point. Based on Special Relativity, matter nor Tachyon matter can travel through space to the speed of light and meet and annihilate. It would take an infinite amount of energy for either to reach the speed of light. Black holes can be renamed as "Neutron Gravity" stars because this is a more accurate description of its state. A neutron gravity star is point of inflection for our universe and the tachyon universe. Our slower than light universe my only see or interact with the gravity part of the neutron gravity star while the faster than light universe only see the antigravity part of the neutron gravity star. A black hole has no mass, no charge, no spin. In which a black hole can't have mass. It is a region of zero curve space (space intercept analogous to a X-intercept on a graph). The escape velocity of the gravity part of the "neutron gravity" star is the speed of light. Reverse is also true for its anti-gravity side. The antigravity part is repulsing objects at the speed of light. Because Tachyon physics is reverse normal physics the repulsive push of the Tachyon black hole is actually the weakest repulsive gravity state there is in the Tachyon universe. In parts of the Tachyon universe there are Tachyon stars that can have a Tachyon gravity field with the repulsive strength of $2(\mathrm{C})$ the speed of light. It's not pulling down velocity like gravity but pushing away velocity. Inside a black hole the two gravity cancel each other out. Any matter that enters into it is instantly annihilated by its tachyon pair and we see that as black hole jets. It contains no mass it has no charge. The black hole can't spin. It exists in a neutral point state where it can't go in one direction. Even though its gravity is neutral like a neutron particle having a neutral charge the slower than light matter is gravitationally attracted to the positive gravity part of the neutron gravity. Likewise, tachyon matter in the Tachyon universe would be affected by the negative pressure antigravity part of the neutron gravity star. Unfortunately, there is no such thing as black holes in the classical sense. When incorporating quantum mechanics in terms of complex conjugates pairs meeting at a point of inflection a black hole is more like a "Neutron Gravity". A spaceintercept simple to the X-intercept on a coordinate plane. This neutral (zero pressure) star is analogous to a neutron particle. Pulsars (neutron stars) are the last state of regular matter stars. A "neutron gravity" star is like a space- 
intercept point region between the universe and tachyon universe. It's a region of neutral pressure space. As the two extremes curve converge their gravities will cancel one another out creating flat space or point of inflection.

\section{Black Hole Jets Explained Based on Annihilation}

A mass warping object and its mirror image can be represented on a complex coordinate plane. You have the real mass $(\mathrm{X}, \mathrm{Yi})$ and its complex conjugate imaginary mass warp (X, -Yi). At extreme gravity levels the mirror image gravities meet at the middle. This middle point is the $\mathrm{X}$ intercept between the two gravities. This X-intercept is the singularity. Because these opposing mass-warp curves meet at X-intercept they will annihilate each other. Twin black hole jets are the result of annihilation of matter and Tachyon matter.

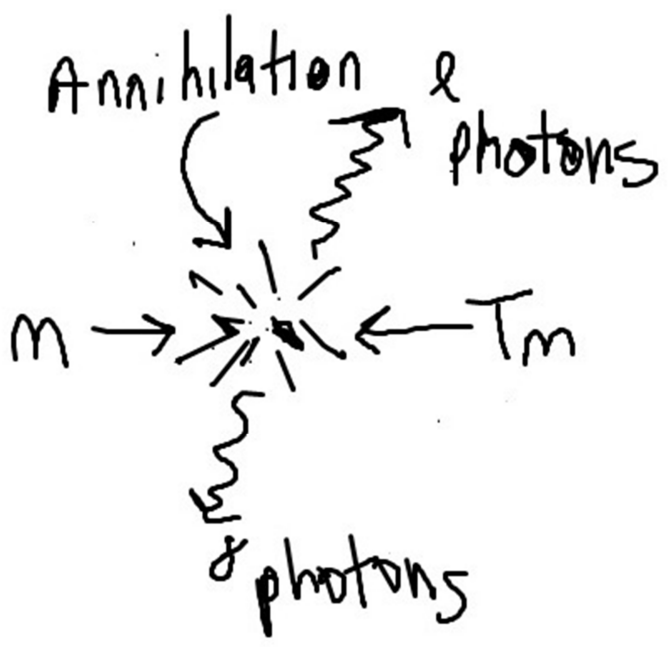

Figure 11. Picture above show matter entering into a black hole (Neutron gravity star) and annihilating its tachyon(T.M.) mirror image partner. Real mass interacting with imaginary mass. The spherical matter will meet with hyperboloid imaginary matter and annihilate and create cylindrical matter (massless complex photon energy)

Based on this GRB model, information that goes into a black hole is recycled as annihilated energy based on Einstein's mass energy conversion equation. All the matter or energy that falls into a black hole is instantly annihilated with its Tachyon mirror image partner. The annihilation energy is complex and half of the energy is spewed out as gamma ray burst from the black hole and the other half of that energy is spewed out of the Tachyon white hole. A black hole is a region of warp space time. No matter exists in a black hole. Initial matter that collapse into a black hole was annihilated by its Tachyon partner and we see that as a GRB. Any new matter that falls into it is annihilated by its Tachyon partner and we see that as black hole jets. Remember spherical matter and Hyperboloid matter annihilate and form cylindrical type flat space. Only massless objects can exist in flat space and must travel at the speed of light. That is why matter is always annihilated and transformed into gamma rays.

Based on this model it appears gamma ray bursts can only be created by the annihilation of matter and tachyon matter at extreme gravities. Even when neutron stars collide they form black hole. It's not the collision of the neutron stars but the gamma ray annihilation from the black hole that produced the GRB. Based on this new model of black holes they have no mass and no magnetic field. Matter can't exist in a black hole to give it a magnetic field. They have no spin. A black hole (neutron gravity star) is a $\mathrm{x}$-intercept (point of inflection) in space-time. Any mass that enters is instantly annihilated by its tachyon partner and produce GRBs. Short GRB is due to the small volume of the colliding dense neutron stars. When you compare object 10 miles across it will annihilate faster than stars 1000 miles across. Longer GBRs are longer because it has more area to annihilate with its tachyon partner. It takes time for the matter \& tachyon matter to meet and destroy each other. Black hole jets are the longest because external matter is feeding the annihilation. The mechanism for all GRB comes from the annihilation of matter and tachyon matter inside black holes (Neutron gravity stars). When the two opposing masses meet they annihilate and form the same type of energy from the big bang

$$
\mathrm{e}(\text { complex })=(\mathrm{mi}+\mathrm{m}) \mathrm{c}^{2}
$$

That energy is complex containing energy and imaginary energy. These complex EM waves will be a composite of visible EM and reverse EM wave structures. We only see the positive part of that energy as GRBs and Tachyon black holes spew out the other half of that total complex energy.

Also It's possible that the mysterious inner core of a Neutron star(Pulsar) may have a small region of zero curved space. In which it's like a black hole but on a smaller scale. Like a black hole matter from its inner layers are falling into the zero region core and annihilating with Tachyon matter and giving raise to the pulsar beams. Because the exiting gamma rays have such dense material to escape from the energy is weak by the time it escapes. The gamma rays exit out as weak radio rays. All that gamma radiation absorbed in the Neutron star layers may be powering its magnetic field. This may explain the connection between pulsar beams and the beams from black holes.

\section{Dark Matter Explained}

The question may arise how does a neutron gravity warp space around it to keep the galaxy in place if its gravity is neutral? Compare a neutron gravity star to a neutron subatomic particle. A neutron particle contains both positive and negative charge that neutralize each other. Apply that to a neutron gravity star and the combined positive and negative space geometries (gravities) cancel each other out. To the surround space that cancellation is also taking place. The positive curvature (gravity) of the Neutron gravity star is warp 
space down while the negative side of the star is warping space up. Producing a flat Euclidean type geometry (vinyl record) encompassing the galaxy. In the context of Einstein Field Equation, a black hole (neutron gravity star) can be described as a cylinder manifold. It is region were the spherically curved matter and Hyperboloid Tachyon matter me forming a cylinder manifold. It is a region of flat space, massless state and everything must travel at the speed of light in that region. The cylinder manifold is a combination of a spherical and Hyperboloid manifold. Meaning in the context of pressure it is holding things in the same place. The neutral pressure is a combination of positive compressing pressure and negative pulling apart pressure. It is a constant state of neutral static scaffolding holding pressure were it holds things in the same place.

Remember that neutral pressure is a combination of negative and positive pressure. It is creating a force that holds things in the same place rather than compressing it or pulling it apart. It is that static hold of neutral pressure flat space that is holding the galaxy intact preventing it from flying apart. Because the neutron gravity star contains both positive and negative curvature the surround matter which is positive curved is attracted to the positive part of the neutron gravity star causing matter to flow into it. The orbital mechanics of a neutron gravity star is different from that of a conventional sun base system.

Neutron gravity star effect on the galaxy can be compared to the hydrodynamics of water. General Relativity depicts space flowing like water in a river flowing. For gravity, a black hole is flowing down like water flowing down a water well. A white hole has the opposite effect where space(water) is flowing up. For neutron gravity stars it is creating the simultaneous effect of space flowing down and up at the same time causing a net zero effect where space stop following. In the water flow analogous to two opposing water streams cancel each other out where the water stop flowing. Technically galaxy is not flowing in space but is at a standstill in space with respect to the gravity water flow analogy. The mass of the galaxy is in this zero flow space. It is the neutral pressure of zero flow space that is holding the galaxy in the same place like scaffolding. Dark matter phenomena are explained by the new interpretation of black holes and its unique gravitational influence on the galaxy. Black holes can be redefined as "Neutron Gravity" Stars. They exert a neutral or static gravity on galaxies acting as scaffolding preventing the galaxy from flying apart.

\section{Dark Energy Replaced by the Dark Wave (Dark Gravitational Sine Wave)}

In looking at the presumed spherical shape of our universe and the imaginary hyperboloid universe from the big bang I found a new wave pattern discovery! They form massive gravity waves on a cosmological scale.
Gravitational waves are defined as the waving of spacetime fabric of the universe. The core idea is that the graph below is the shape of the gravity waves from the big bang. They're not the supposed ripple gravity waves in the cosmic microwave background radiation (CMB). The expansion and acceleration of the universe is actually a Cosmic gravity "Dark wave". The universe is expanding within the Dark Wave, going for a ride. The trajectory of mass is governed by these colossal gravity waves. Embedded in the wave so as the wave expand our universe expand.

In big bang cycles the universe alternates between spherical shapes (matter universe) and FTL hyperboloid shapes (imaginary mass universe) in a wave pattern. I've use a $2 \mathrm{~d}$ wave to represent the $3 \mathrm{~d}$ cosmological gravity wave of the alternating universes in big bang cycles. One universe is made of matter and the next cycle is antimatter.

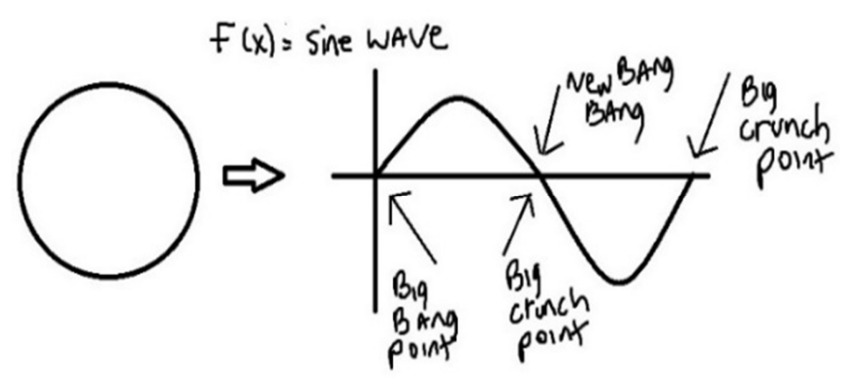

Figure 12. The graph represents the sine wave of the universe from the big bang to the big crunch. Top semicircle represent the universe from big bang to big crunch. The Big crunch is also an inflection point were in the next big bang the universe will have a trough maximum width before its big crunch again into a new singularity point big bang cycle. Using sine waves and not a probability wave function of the distribution geometry of space-time based on certain quantum mechanical fluctuations is the way to model the universe.

In a reverse cycle were imaginary mass is created first It starts as a hyperboloid universe and in the next big bang it will be a spherical universe. The graph below shows a universe propagating first as a Hyperboloid universe of Tachyon matter and then a Hyperboloid universe of antiTachyon matter in alternative sine wave big bang cycles.

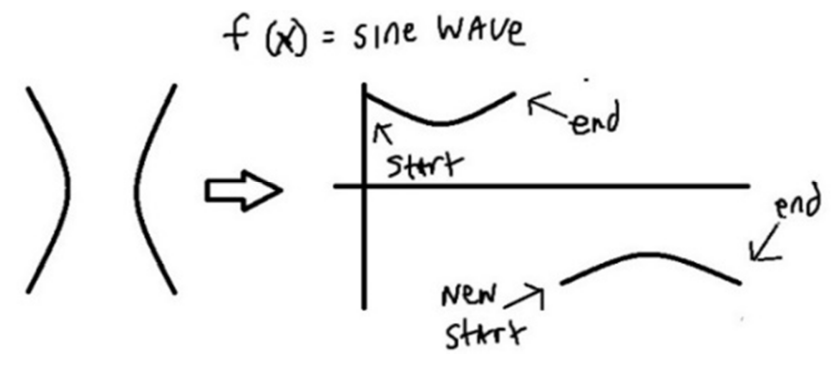

Figure 13. The hyperboloid Tachyon universe can be represented as a discontinuous sine wave function. One half of the hyperbolic parabola represents a Hyperboloid universe from its start (big ring beginning) to its minimum width and then its open end (final big ring state). The other half of the hyperbolic parabola represents the next Hyperboloid universe in its new big bang cycle. One arc of the hyperbolic is 0 to 180 degrees and the other arc is 181 to 360 degrees. Together they represent a full hyperbolic 360degree open circle which is a reverse mirror image of the 360 degree closed circle. 
When determining the geometry and final state of the universe you must use a sine wave function and not probability wave function of the distribution geometry of space-time based on certain quantum mechanical fluctuations.

Visible matter universe is in de sitter space and the Tachyon universe is in anti-de sitter space. The two states are quantum entangled operating as one space. The hyperboloid universe has a finite beginning and ending coinciding with the big bang and big crunch of our universe. Just as our universe started at a finite point the Tachyon universe started at a finite Ring. Just as the universe will collapse into a big crunch point the tachyon universe will stop at a finite big ring. Both universes are quantum entangled and must balance out to zero.

Based on the cosmological wave pattern the universe propagates as a wave started by the big bang. As the universe waves from the big bang point it is approaching the crest (maximum) point of the wave and then the big crunch. Approaching the crest part of the universe wave is what we interpret as the expansion and acceleration of the universe. Our visible universe will end in a big crunch as the result of being part of a wave. The "Dark Wave" model will give the wavelength, frequency, propagation speed of the wave and tell us the energy of the waving universe and how big it can expand and when the universe will end at the big crunch. The Dark Wave model can be compared to astronomical observations.

The complex energy responsible for the matter and Tachyon universe came from the $\mathrm{x}$-intercept. Each went off into equal and opposite directions from the central $\mathrm{x}$-intercept point. Matter expanded in the upper quadrant and the Tachyon matter went to the lower quadrant. The singularity point of matter wasn't the actual singularity point of creation. The matter singularity point we normally think of is the beginning of the spherical universe. It's like a point on the south pole. The Tachyon universe also had a mirror image started point. It wasn't a singularity point but a Ring where it was the base of a hyperboloid ring. First we had the $\mathrm{x}$ intercept point of complex energy and then transformed into pair production of matter and Tachyon matter. Matter went in the upper quadrant and Tachyon matter went in the lower quadrant.

Overtime the universe will accelerate as close to the speed of light as it can get. It is predicted the universe will accelerate to $99.99 \%$ of the speed of light while the Tachyon universe will slow down to just above the speed of light. Both are quantum entangled and acting as one universe and balancing each other out. As our universe reach its maximum width the Tachyon universe will reach its minimum width and beyond that things will reverse. The quantum entanglement is responsible for the acceleration and expansion of Our universe. Our universe singularity point started at $\mathrm{V}=0$ while the Tachyon "Big Ring" started at $2(\mathrm{C})$. Over time their balancing each other out. In the middle state the universe will have accelerated to virtually the speed of light while the Tachyon universe will decelerate to almost the speed of light. Beyond the middle growth point the universe will contract and slow down to $\mathrm{V}=0$ while the Tachyon universe while expand to $2(\mathrm{C})$ in its final state. Our universe will contract to a big crunch while the Tachyon universe will expand to a finite big ring. The total energy between the universe and Tachyon universe will balance out to zero like in any quantum entangled system.

The dual big bang based on the equation below produced the two complex conjugate "Dark Waves" that are quantum entangled.

$$
\mathrm{e}(\text { complex energy })=(\mathrm{m}+\mathrm{mi}) \mathrm{c}^{2}
$$

As our universe expands overtime the hyperboloid universe contracts were they balance out due to quantum entanglement. From beginning to end the two universes are connected and operate as one universe system. Those restrictions of quantum entanglement with the Tachyon universe lock in the uniformity of the visible universe.

\section{Imaginary Universes (Zi Universes)}

On the complex coordinate plane, we see universes and Tachyon universes start and end on real points on the number line. They are universes of mass. Our universe has spherical mass and Tachyon universe have Hyperboloid shape proton type particles. In theory there should exist a third type of universe. A universe with a cylinder shape. It's would be a pure massless universe where the atoms are massless. It has no beginning nor end. It's an eternal universe. Just as mass universes start and end on the real number line the cylinder universe exist purely on the imaginary $\mathrm{Zi}$. Imagine its start and end state being the same point on the $\mathrm{Zi}$ axis. There are an infinite number of different cylindrical type universes like a vertical multiverse like the physical mass multiverses. Just as you have 3 basic types of geometries there exist 3 basic types of universes. Positive and negatively curved universes exist on the $\mathrm{x}, \mathrm{y}$ real number axis while the cylindrical universes exist in the imaginary $\mathrm{Zi}$ axis.

Just as universes can exist on the real $x, y$ real number exists(sphere, hyperboloid universes) the cylindrical universes exist on the imaginary $\mathrm{Zi}$ axis. Its start and end point is an imaginary point compared to real number points for the physical universes. Cylinder universes would be purely massless universes. Just as protons or hyperboloid protons make up mass universes the imaginary cylindrical universes would have massless atomic structures. An infinite number of different cylindrical universes can exist on the imaginary axis like different parallel universes can exist on the real $\mathrm{x}, \mathrm{y}$ axis. Image below on $2 \mathrm{~d}$ graph show sine wave propagation of universes on $\mathrm{x}$ axis. Located on the vertical imaginary yi axis are the infinite cylinder universes. 


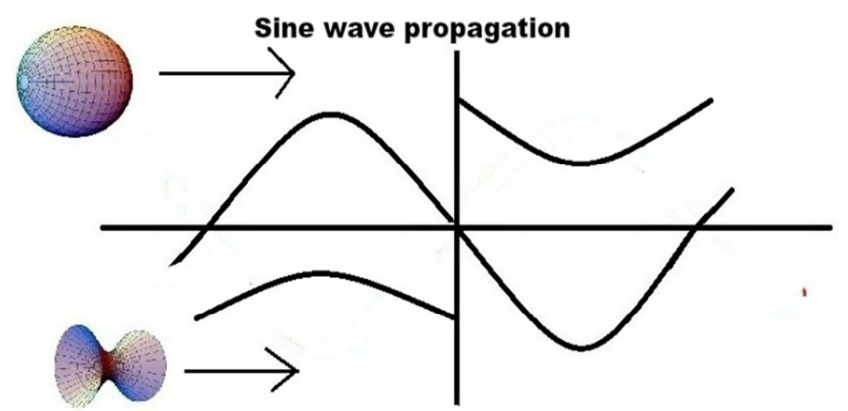

Waves can propagate to infinity

Figure 14. The big bang created matter and imaginary matter pair universes that started as cosmological gravity waves. Keep in mind the $2 d$ graph is a simple sine wave representation of the $3 d$ universes as they follow in time. They are complex conjugate pairs and quantum entangled. Complex conjugate math is used to represent quantum entanglement. Our universe consists of continuous sine waves while our complex conjugate partner consists of discontinuous sine wave. We see that the universe and Tachyon universe are reverse in every way. Combine them together and they balance out to zero. They propagate as parallel wave pairs from the initial big bang of matter-Tachyon matter. They can wave to infinity and that represents the multiverse. The waves can vary in wavelength, frequency, energy, quantum mechanics, etc. and that will coincide with different physics unique to that parallel universe. For example, on a different universe atoms may consists as actual strings but not exactly like the string theory we think of. Imagine a sphere particle that isn't solid but consist of a string that spiral in $3 d$ and make a sphere. That would be a closed string. Likewise, for Hyperboloid particles they not consist of solid particles but one string spiraled in $3 d$ that is shaped like a Hyperboloid. This would be an open string. Who knows this may be the subatomic structure of particles in our universe. Image below gives example of particle-strings.

It's interesting to note that the product of two complex numbers is a real number. Both matter and Tachyon matter came from a real number singularity point. Overtime matter can be presented as complex universes and Tachyon matter as its complex conjugate universes. The product of those complex numbers are real numbers. However, for the cylinder universes they are on the imaginary axis.

\section{From I.M. Lambda-CDM to String Theory}

Over time the universes are propagating like waves through alternating periods of real matter and imaginary matter creation cycles to infinity. Our universe represents the continuous sine wave and the Hyperboloid universe is represented by the discontinuous sine wave. The multiverse sine wave universe model of the I.M. Lambda-CDM model coincides with the multiverse model of String Theory. However, it's not an exact match because String Theory predicts Gravitons as open strings while the other fundamental particles as closed strings. Unlike String Theory this theory identifies slower than light mass particles correlating to closed strings and all FTL hyperboloid open end particles correlating to open strings. In which the I.M. Lambda-CDM is describing a new form of String Theory. The following shows how strings are particles based on a new concept called $3 \mathrm{~d}$ winding number theory:
Spiral String Theory
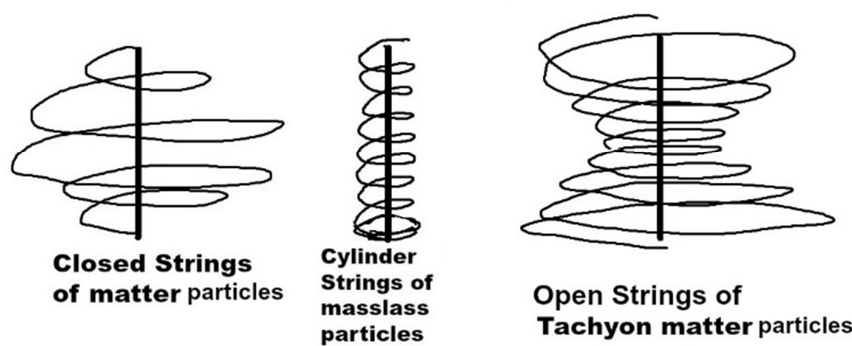

Open Strings of

Tachyon matter particles

Figure 15. $3 d$ winding number theory is an extension of conventional winding numbers. The number of times string winds around a circle is called the winding number, and that is also quantized (as it must be an integer). Rather than using a flat $2 d$ surface of a central point and making curved circles around it represented as integers (quantized states) extend that concept 3 dimensionally. In which the $2 d$ central point is extended as a central $3 d$ pole with a finite length. The 1-dimensional strings are winding in 3-dimensions around that finite pole. From pole to pole curve around it making winding numbers in integers (quantized states). Depending on the space curve geometry the path of the string will take straight lines on the curved surface. For positive curvature it will wind like a sphere from pole to pole. Negative curve space it will wind like a Hyperboloid. For flat space it will wind like a cylinder. In so doing the strings literally takes the shape of particles! $3 d$ winding number theory is the unification of string theory and particle physics! From a mathematical perspective this $3 d$ winding number can represent as a sine wave. Smaller waves on the end and big waves at the center area. The breakthrough is that wave pattern matches a spherical standing wave. The electron is a spherical standing wave system. A Tachyon particle standing wave would have bigger waves in the outer ends and smaller waves in the middle area. The standing wave can move were it is analogous to vibrating strings. We see the transition from $3 d$ winding number theory for string theory and its connection to traditional QM spherical standing wave systems. Figure 15 may be literal image of particles were their not solid curves surfaces but consists of spiral $3 d$ strings that take on the shape of point like particles.

Wikipedia defines String theory as a theory that holds that the fundamental constituents of subatomic particles, including the force carriers (e.g. Leptons, photons, and gluons), are all composed of a one-dimensional string of energy that takes on its identity by vibrating in different modes and/or frequencies. Traditional 1-dimensional string theory is based on a flat $2 \mathrm{~d}$ winding number theory. This paper goes a step further. By using 1-dimensional strings that wind in $3 \mathrm{~d}$ based on $3 \mathrm{~d}$ winding numbers causes strings to resemble the physical structure of particles. It is that stringparticle structure that will give a more accurate real world description of vibrating strings. Because of the string-particle structure we may only need 4-dimensions of space-time and not 11 dimensions.

We see the particle-string duality of matter. A $3 \mathrm{~d}$ spiral string can take on the overall shape of a particle. Because we can't see the surface of particles we assume their smooth continuous surfaces but in fact their quantized spiral strings in the form of a point like particle. This is analogous to the particle-wave duality of matter. In quantum interaction it can either be interpreted as point like particles or $3 \mathrm{~d}$ winding string interactions. For example, a matter and antimatter collide to form gamma ray photon from a particle point interaction interpretation. Now imagine those interactions as $3 \mathrm{~d}$ spiral springs. Matter and antimatter is represented as 
spiral strings rotating in opposite directions. Those two oppositely rotated spherical spirals interact and form a cylindrical massless $3 \mathrm{~d}$ spiral string. That cylindrical string represents the gamma ray after annihilation. The interaction is spread among the spiral strings rather than point like interaction.

Next-gen string theory called "Spiral string theory" is based on the idea that particles may not be solid sheet particles but 3-d spiral strings that contour like particles. In which $3 \mathrm{~d}$ winding quantum numbers can form $3 \mathrm{~d}$ shaped particle spheres (closed strings) and Hyperboloids (open strings). This new idea is represented as $3 \mathrm{~d}$ winding number theory. In which the strings are particles. A spherical spiral string is a closed string while a Hyperboloid particle is an open string. The physics and vibration of the string can be interpreted based on traditional string theory. The quantum leap idea isn't that string represent particles it is strings are particles! The strings 3 dimensionally spiral and contour to the shape of particles according to space curve geometry of the universe. In which particles aren't curved solid sheets of matter but consist of $3 \mathrm{~d}$ winding number spiral strings that contour to the shape of a particle based on the type of space time geometry it is in. Matter and antimatter can be based on the string spiraling clockwise or counterclockwise in forming an overall spherical shape. Also Tachyon particles and antiTachyon particles spiral in opposite clockwise paths. For matter and Tachyon pair they also spiral in opposite directions. For massless particles they are spiral cylinder strings. They too can be close wise and counterclockwise cylinder spirals. The number of winds would give the quantized state of the massless particles. If the spiral rotates in one direction from pole to pole, then it represents (for example) EM waves (photons) but if it spirals in other direction then it represents the reverse EM Tachyon wave structure. You see with $3 \mathrm{~d}$ winding theory the strings take on the physical form of particles. That lead me to believe that strings are literally particles! $3 \mathrm{~d}$ wing number string theory is next-gen string theory or "Spiral string theory".

\section{3d Winding Number Theory}

At the heart of Spiral string theory is $3 d$ number theory. When you take conventional winding number theory you have a central point and it represents the total number of times that curve travels clockwise around that point. Conventional string theory is based on this. What if this $2 \mathrm{~d}$ winding number theory is simply looking at the top or bottom view of a $3 \mathrm{~d}$ winding number theory. In other words, what if the point in $3 \mathrm{~d}$ represents a finite long pole. Rather than make curves around a flat point you're making those same quantized curves in $3 \mathrm{~d}$ from pole end to pole end. The total number of times that curve travels around this pole vertically from one end to the other is represented as whole integers (quantized states). Based on the 3 types of space curve geometries $(+, 0,-)$ you have 3 basic types of $3 \mathrm{~d}$ spiral strings. In one case the 1-dimensional strings can spiral around the finite pole in a Spherical pattern. The other case is where the 1-dimensional string spiral from point to pole in a Hyperboloid pattern. For flat space it will spiral around the pole from end to end a finite number of times (a certain amount of integers) like a cylinder. Examples of Cylindrical spiral strings are massless photons, gravitons and any other mass particles. The photon and Graviton can have different $3 \mathrm{~d}$ winding numbers. Just like with 1-dimensional strings based on $2 \mathrm{~d}$ winding number theory where vibration modes of the string that represent different particles with different masses the same applies to 1-dimensional strings using $3 \mathrm{~d}$ winding number theory. At the lowest mode were $m=0$ and spin is 2 represents the graviton. It reduces gravity at low energies. The major breakthrough is that conventional string theory used a $2 \mathrm{~d}$ winding number theory while spiral string (S string) use $3 \mathrm{~d}$ winding number theory. When $3 \mathrm{~d}$ winding number theory is applied the shape of the 1-dimensional string makes the spherical particles in our universe and the Hyperboloid particles in the Tachyon universe. In other words, the $3 \mathrm{~d}$ winding strings taking on the shape of particles. It leading to the idea that $3 \mathrm{~d}$ wound 1 -dimensional strings are the actual particles!

Spiral String theory will give a more practical view of the Graviton in quantum gravity theory. Another way to visualize the Graviton in quantum gravity is to imagine a gravity well that stretches down to a black hole point. Superimpose that gravity well to an electromagnetic spectrum. The top part of the gravity well is like radio waves of the EMS. The bottom part of the gravity well representing the neutron star and black hole is like the Gamma ray frequency part of the EMS. Based on this correlation of a gravity well to the EMS the gravity well can be viewed as a "Gravity wave spectrum". Just as the photon is the quantum of the EMS the Graviton is the quantum of the "Gravity wave spectrum". The following illustrations further highlight the correlation:
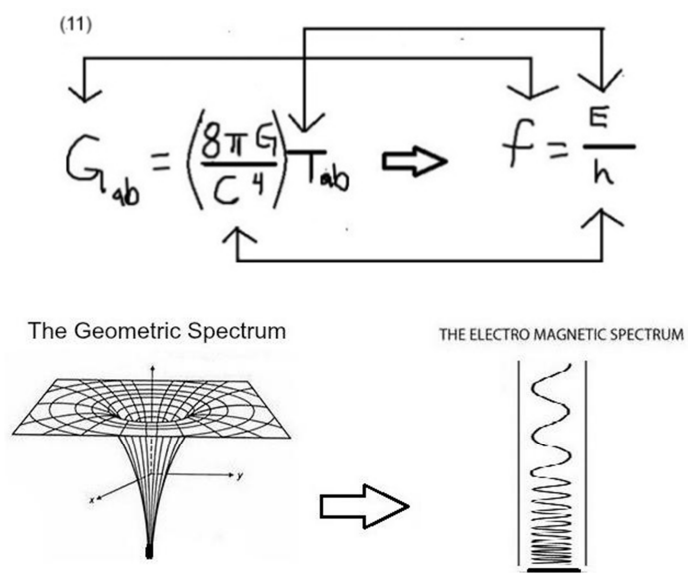

Figure 16. $F$ is the frequency of curved space-time where frequency is interpreted as the relationship between the warpage of space and time flow. For example, in stronger gravity fields time flows more slowly but in weaker gravity fields time flows faster compared to objects in stronger gravity fields. Frequency of curved space-time would be the rate of time in the frequency or gravity field strength of curved space. This frequency of curved space-time corresponds to the pure geometry side of Einstein's Field Equation. E is gravitational energy were that corresponds to the energy-stress-momentum tensor in Einstein's Field Equation. The Planck constant of curved space is denoted $h$ where it is a physical constant that is the quantum of action of 
gravity. It is central in quantum gravity. This Planck constant of curved space corresponds to the constants in Einstein's Field Equation. The geometric nature of gravity is interpreted as a spectrum like the EM spectrum. The Graviton is the quantum of the Geometric spectrum (Gravity wave spectrum) like the photon is the quantum of the EM spectrum. The correlation between gravity and EM also work for repulsive gravity where you use imaginary values for gravitational energy (Ei). Please note this is an imaginary value is used in the context of Relativity equations were slower than light matter has real number values and FTL matter or energy have imaginary values. Meaning it doesn't apply to the complex coordinate plane of QM wave functions where both matter and Tachyon matter are represented as complex conjugate pairs. A consequence of this concept is that Special Relativity can be quantized were time dilation for objects traveling close to the speed of light can be interpreted as the frequency of time dilation. In which at Relativistic speed the rate of time slows down while the mass becomes larger. This behavior is a frequency similar to a EM wave frequency. This new Planck Constant for Special and General Relativity is essentially the Quantum in Quantum Relativity. In which for Quantum Special Relativity "The Electrodynamics of moving bodies" can be renamed as the "Electrodynamic Spectrum of moving bodies "because time dilation can be expressed as a frequency like EM wave frequencies. Gravity can be expressed as the frequency of space-time curvature.

\section{S-Theory Cosmology}

The imaginary mass Lambda-CDM can be called the "Spiral String cosmological model" or "S-Theory cosmology". The winding quantum numbers of string theory can form into the shape of spherical close particles and open end Hyperboloid particles. In which strings don't represent particles, strings are particles! This is a unification of particle physics and String theory. They are one and the same. Surface topology of a particle isn't a smooth continuous curved surface but is a string that spiral in $3 \mathrm{~d}$ to make a hollow sphere shape. In which Spherical or hyperboloid particles comprise of winding quantum numbers that form those $3 \mathrm{~d}$ shapes of spheres or hyperboloids. Imagine "M" theory and modifying it into "Spiral String theory". Just as M-theory was a revolution in string theory the Spiral String theory is that next and final form of String theory. Early string theory predicted the Tachyon particles. Based on this paper that was actually corrected. If we have a big bang pair production of closed spiral spherical strings(particles) and open spiral Hyperboloid particles(Tachyon) then it perfectly matches the early prediction of string gas cosmology where we have a dual universe where we have a universe that expands (visible universe) and a universe that contracts (Tachyon universe).

String gas cosmology predicts a dual universe and that duality perfectly coincides with the matter and the FTL tachyon universe based on a big bang matter and Tachyon matter pair production. Our universe is expanding following a spherical path while the tachyon universe is contracting taking a Hyperboloid path and their both quantum entangled operating as one system. Like traditional string gas cosmology that has a Hagedorn phase so does Spiral string theory cosmology. We see a T-duality between the universe and Tachyon universe. Once M- theory is modified to have the new spherical and Hyperboloid winding quantum numbers it will revolutionize the field of string theory and finally make it practical and testable.
While M-Theory has flat sheet type cosmological branes based on 1-dimensional vibrating strings the Spiral String theory (based on $3 \mathrm{~d}$ curved spiral strings) will give rise to more $3 \mathrm{~d}$ recognizable shapes. For example, our spherical curved universe and the Tachyon universe can be interpreted as branes in de sitter and anti-de sitter space. Parallel universes can have different types of 3d "Spiral String base cosmological branes". The visual interpretation of these branes are the cosmological gravity sine waves from figure 14 . In which the sine waves are like cosmological vibrating strings! Spiral string theory(S-Theory) like M-Theory should give all the possible combination of Spiral strings to give us all the possible combination of the multiverse. From conventional string theory it used 1 dimensional vibrating strings and had 10 to the 500 possible combination. We thought that was a big number. Now imagine how many Spiral String or 3 dimensional vibrating string combination there are!

Spiral string cosmology (S-Theory cosmology) contains parallel universes like traditional string theory. Imagine for a moment if our dreams show us those parallel universes in real time. The way that could be possible is by Einstein's Spooky action at a distance. You're seeing instantaneously in real time something that is happening an unbelievable distance away. Parallel universes bring up the question of religion. In which are their parallel heavens? No longer the debate of science vs creation but multiverse science vs multiverse creation. In a way the parallel universe or Heavens make up one overall universe or Heaven if you interpret them as a supercomputer. Just as parallel computers operate as one supercomputer the parallel universe act as one super universe or superverse. The same applies to Heaven. It's still one God over everything but in a supercomputer format. Who knows parallel Heavens may exist on the imaginary $\mathrm{z}$ axis while the universes exist on the real number $x, y$ axis. For the imaginary z-axis universes imagine the starting point and ending point being the same point. It has no beginning or end but is eternal. Those parallel universe lay on the vertical imaginary $\mathrm{Zi}$ axis while spherical and Hyperboloid universes lay on the real $x, y$ axis. The shape of the universe would be cylindrical in relation to our de sitter space universe and the anti-de sitter space Tachyon universe. This cylinder multiverse universe state is referred to as the "God universe" because those parallel universes have no beginning or end but is in a constant state unlike our temporal spherical universe and Hyperboloid universe. Just as our universe is positively curve and Hyperboloid universe is negatively curved with finite time amounts the cylinder universe has no beginning or end. If universes existed on the imaginary axis, then its Start and finish would be one and the same. These are eternal parallel universes that exist on the imaginary z-axis. Even though parallel universes are a mind boggling distance away Imagine a Tech future of parallel universe travel and multiverse computer search engine based on Einstein's spooky action at a distance physics technology and Tachyon EM drive. A time in computer social networking you can be friends with your parallel selves. The next big thing in physics will be multiverse research study and the next big in the church will be believers praying over their parallel selves 
and dreams. Their Bibles and what faith is, is different from our bible but parallel in goodness. The battle between good and evil is also on a parallel universe scale. Their Book of Genesis to Book of Revelation is different from our bible. The church can pray over selves that their parallel selves do what the bible says in their own universe. The processing power of the supercomputer universe is infinite where it's processing speed can be described as a "Uniflop" like actual supercomputer processing speed is described as Teraflop or Petaflop for example. The supercomputer universe multiverse theory can be called the "Superverse" were all the parallel universes act as one supercomputer like the individual computers of a supercomputer work together as one Teraflop or exaflop supercomputer. Parallel universe theory isn't new but S-Theory cosmology (formerly called the I.M. Lambda$\mathrm{CDM}$ ) will make it widely accepted like the world transitioned from believing the earth is the center of the solar system to the sun being the center of our solar system from Galileo work. The I.M. Lambda-CDM model can be called the "Spiral String cosmological model (S-Theory Cosmology)" because it was actually describing a new form of String theory. In which Spiral String Theory (S-Theory) is the next breakthrough beyond M-Theory. Just as M-Theory unified 5 different string theories into one theory the S-Theory unifies particle physics and M-theory into one. In which particles are 1-dimensional strings based on $3 \mathrm{~d}$ winding number theory. The $3 \mathrm{~d}$ wound 1dimensional strings take on the shape of particles!

\section{Conclusions}

By replacing a matter and antimatter big bang pair production with a matter and Tachyon matter pair production it fully explains the effects of Dark Energy, Dark Matter and a host of other cosmological phenomena. Throughout the paper we saw that by incorporating imaginary mass into the Lambda-CDM model it was actually describing a new form of string theory called "Spiral string" theory. This new form of string theory can give a more accurate description of quantum gravity. Computer modeling and Independent verifications of the Tachyon EM experiment (building Tachyon transmitters or receivers) will prove the validity of this new Spiral String cosmological model.

\section{References}

[1] P. Kroupa, B. Famaey, K.S. de Boer, J. Dabringhausen, M. Pawlowski, C.M. Boily, H. Jerjen, D. Forbes, G. Hensler, M. Metz, "Local-Group tests of dark-matter concordance cosmology. Towards a new paradigm for structure formation" A\&A 523, 32 (2010).

[2] Andrew Liddle. An Introduction to Modern Cosmology (2nd ed.). London: Wiley, 2003.

[3] Camille M. Carlisle, Planck Upholds Standard Cosmology, Sky \& Telescope, February 10, 2015

[4] a b Dodelson, Scott (2008). Modern cosmology (4. [print.]. ed.). San Diego, CA [etc.]: Academic Press. ISBN 9780122191411.

[5] Frieman, Joshua A.; Turner, Michael S.; Huterer, Dragan (September 2008). "Dark Energy and the Accelerating Universe". Annual Review of Astronomy and Astrophysics 46 (1): 385-432. arXiv: 0803.0982. Bibcode: 2008ARA\&A. 46..385F. doi:10.1146/annurev.astro.46.060407.145243.

[6] Kovac, J. M.; Leitch, E. M.; Pryke, C.; Carlstrom, J. E.; Halverson, N. W.; Holzapfel, W. L. (19 December 2002). "Detection of polarization in the cosmic microwave background using DASI". Nature 420 (6917): 772-787. arXiv: astro-ph/0209478. Bibcode: 2002Natur.420..772K. doi: 10.1038/nature01269. PMID 12490941.

[7] Collaboration, Planck; Ade, P. A. R.; Aghanim, N.; Arnaud, M.; Ashdown, M.; Aumont, J.; Baccigalupi, C.; Banday, A. J.; Barreiro, R. B.; Bartlett, J. G.; Bartolo, N.; Battaner, E.; Battye, R.; Benabed, K.; Benoit, A.; Benoit-Levy, A.; Bernard, J. -P.; Bersanelli, M.; Bielewicz, P.; Bonaldi, A.; Bonavera, L.; Bond, J. R.; Borrill, J.; Bouchet, F. R.; Boulanger, F.; Bucher, M.; Burigana, C.; Butler, R. C.; Calabrese, E.; et al. (2015). "Planck 2015 Results. XIII. Cosmological Parameters". arXiv: 1502.01589 [astro-ph.CO].

[8] Spergel, D. N. (2015). "The dark side of the cosmology: dark matter and dark energy". Science 347 (6226): 1100-1102. Bibcode: 2015Sci...347.1100S. doi:10.1126/science.aaa0980. PMID 25745164.

[9] a b Table 4 on p. 31 of Planck Collaboration. "Planck 2015 results. XIII. Cosmological parameters" (PDF). Retrieved 2015-02-18.

[10] Appendix A of the LSST Science Book Version 2.0. 